\title{
Chemcomm
}

Chemical Communications

rsc.li/chemcomm
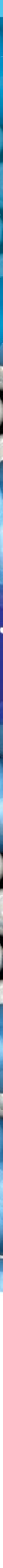
Check for updates

Cite this: Chem. Commun., 2018, 54,8316

Received 1st June 2018,

Accepted 22nd June 2018

DOI: $10.1039 / \mathrm{c} 8 \mathrm{cc} 04380 a$

rsc.li/chemcomm

\section{Periodic and nonperiodic chiral self-assembled networks from 1,3,5-benzenetricarboxylic acid on $\mathrm{Ag}(111) \dagger$}

\author{
Joshua Lipton-Duffin, (D) *ab Maryam Abyazisani (D) ${ }^{a}$ and Jennifer MacLeod (D) *ab
}

\begin{abstract}
Surface-catalyzed reactions provide a versatile route to synthesizing new 2D materials. Here, we show that the statistical nature of an activated reaction can lead to a diversity of intermolecular bonding motifs through partially-reacted molecules. With increasing annealing, we observe different periodic, well-defined phases of 1,3,5-benzenetricarboxylic acid on $\mathrm{Ag}$ (111), where the surface structure in each case is defined by the degree of deprotonation of the carboxylic groups. Over a wide range of deprotonation levels ( $50 \%$ to near $100 \%$ ), we observe a granular alloy comprising two distinct phases in a continuous network. This ordered phase lacks well-defined translational symmetry, is stabilized through both intermolecular interactions and epitaxy, and demonstrates a design approach to creating non-crystalline phases by capitalizing on the chemical diversity of partially reacted molecules on a surface.
\end{abstract}

Molecular self-assembly at surfaces provides a rapid, scalable route to $2 \mathrm{D}$ films with tailored chemical, electronic and topological characteristics. ${ }^{1,2}$ This level of nanoscale control is a key enabling capability for next-generation applications in sensing, electronics and energy conversion and storage, ${ }^{3,4}$ and direct observation of these molecular systems through scanning tunnelling microscopy (STM) has provided a depth of fundamental understanding of the factors that drive molecular self-assembly at surfaces. ${ }^{5,6}$ A core approach to generating this understanding has been a focus on model molecular systems, such as 1,3,5-benzenetricarboxylic acid (trimesic acid, TMA). TMA is a $C_{3}$-symmetric hydrogen bonding unit that tends to form strong $\mathrm{R}_{2}^{2}(8)$ cyclic dimers or somewhat weaker $R_{3}^{3}(12)$ trimeric associations in $2 \mathrm{D}$ assemblies. ${ }^{7}$ On unreactive surfaces, such as graphite, graphene, or $\mathrm{Au}(111)$, these bonding motifs define the long-range molecular arrangements. $^{8-12}$ On more reactive surfaces, such as copper,

\footnotetext{
${ }^{a}$ School of Chemistry, Physics and Mechanical Engineering, Queensland University of Technology (QUT), Brisbane, 4001 QLD, Australia. E-mail: josh.liptonduffin@qut.edu.au, jennifer.macleod@qut.edu.au

${ }^{b}$ Institute for Future Environments, Queensland University of Technology (QUT), Brisbane, 4001 QLD, Australia

$\dagger$ Electronic supplementary information (ESI) available: Additional STM images, O1s XPS, details of DFT calculations. See DOI: 10.1039/c8cc04380a
}

the $-\mathrm{COOH}$ groups can catalytically deprotonate to $-\mathrm{COO}^{-}$, creating the possibility for ionic hydrogen bonding and metalcoordinated bonding. ${ }^{13-17}$ Similarly, partial deprotonation occurs when TMA is solution-deposited onto $\mathrm{Cu}^{-}$or Ag-modified $\mathrm{Au}(111) \cdot{ }^{18,19}$ At room temperature on $\mathrm{Ag}(111)$, TMA self-assembles through hydrogen bonding, and annealing to $420 \mathrm{~K}$ produces a partially-deprotonated TMA phase comprising dense planar dimers stabilized by ionic hydrogen bonds; ${ }^{20-22}$ a theoretical model for this system suggests that the molecules are one-third deprotonated. ${ }^{20}$

Annealing TMA/Ag(111) to higher temperatures produces a range of phases with different appearances, ${ }^{23}$ but a detailed exploration of the structure and chemistry of these phases has not yet been made. Here, we use a combination of STM and X-ray photoelectron spectroscopy (XPS) to show that annealing to temperatures between $460 \mathrm{~K}$ and $490 \mathrm{~K}$ produces a nonperiodic phase that can accommodate a range of deprotonation states of TMA. In recent years, disordered and quasicrystalline 2D selfassembled phases have gained attention, largely for their fundamental interest. ${ }^{24,25}$ In these investigations, disorder has been, e.g., structural, arising from multiple available bonding geometries $^{26}$ or compositional, arising from bicomponent mixing. ${ }^{27}$ Recent work also showed that the presence of multiple vertex geometries in a metal-organic network can stabilize quasicrystallinity. ${ }^{28}$ In the present case, we find that after annealing TMA on $\mathrm{Ag}(111)$, a locally-crystalline phase without long-range order forms due to the range of chemical states of the TMA precursor, which is accommodated by the seamless integration of two distinct epitaxially-constrained assembly motifs. We hypothesize that the structure incorporates both metal-coordination and hydrogen bonding. This phase elucidates the potential for chemical and topological complexity in a molecular assembly formed from a single, simple precursor molecule.

All experiments were performed in an ultrahigh vacuum system (ScientaOmicron $\mathrm{GmbH}$ ) incorporating both a twin-anode X-ray source (DAR 400) and analyser (iSphera) and a variabletemperature scanning tunnelling microscope (VT-AFM/XA). A (111)-oriented single-crystal Ag substrate (Mateck, GmbH) was cleaned with repeated $\mathrm{Ar}^{+}$sputter/anneal cycles, and sample 
cleanliness was verified with XPS. TMA (Tokyo Chemical Industry) was evaporated from an organic effusion cell (MBE Components OME40) held at $425 \mathrm{~K}$. Samples were annealed at the specified temperatures for five minutes. STM images were processed with the WSxM software. ${ }^{29}$

Following the deposition of $\sim 1$ monolayer (ML) of TMA onto a room-temperature (RT) Ag(111) substrate, phases typical of hydrogen bonded self-assembly were observed (see ESI $\dagger$ for STM). ${ }^{20,21}$ XPS confirmed that the TMA molecule was intact (Fig. 1a). This is evident in the C1s core level, which contains a primary spectral contribution associated with the benzene ring at $\sim 284.8 \mathrm{eV}$, and a smaller component associated with the carboxylic groups at $\sim 288.9 \mathrm{eV}$, in a $2: 1$ intensity ratio. ${ }^{20}$ The $4.1 \mathrm{eV}$ spacing between these two peaks is expected for intact $\mathrm{TMA}^{20}$ and is corroborated by the O1s region, which shows near-identical intensities for the $-\mathrm{OH}$ and $=\mathrm{O}$ contributions (see ESI $\dagger$ ). Annealing the sample to $430 \mathrm{~K}$ produced a dimer phase, similar to one identified in previous studies as an ionic hydrogen-bonded assembly (see ESI $\dagger$ for STM). ${ }^{20,21}$ XPS revealed that this phase corresponds to a partial deprotonation of the carboxylic acid groups, evidenced by the appearance of a spectral contribution at $\sim 288 \mathrm{eV}$ (Fig. 1b), which is consistent with

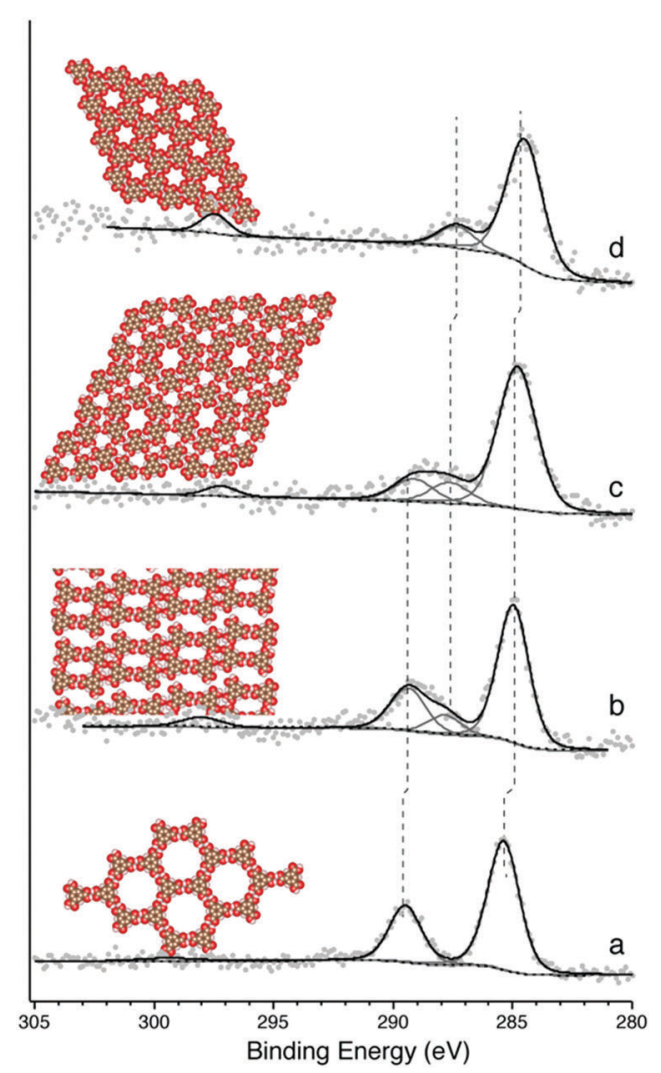

Fig. 1 Schematic structure and C1s core level spectrum for different TMA phases observed on $\mathrm{Ag}(111)$. The XP spectral component at $\sim 284.8 \mathrm{eV}$ arises from the aromatic core of the TMA, the component at $289 \mathrm{eV}$ is associated with $-\mathrm{COOH}$ and the component at $288 \mathrm{eV}$ is associated with $-\mathrm{COO}^{-}$. The feature at $298 \mathrm{eV}$ is thought to be a shake-up satellite. (a) Chicken wire structure, (b) dimer structure, (c) discrete pinwheels, (d) knitted pinwheels.
$-\mathrm{COO}^{-} ;^{17,20}$ again, this observation is corroborated by the $\mathrm{O} 1 \mathrm{~s}$ region (see ESI $\dagger$ ). The spectral intensity observed in C1s suggests $\sim 25 \%$ deprotonation, which is close to the $33 \%$ deprotonation level of the proposed model for this system. ${ }^{20}$

Annealing to higher temperature increases the average deprotonation of the TMA. Fig. 1c shows the C1s region after annealing to $475 \mathrm{~K}$ : at this point, the TMA is $50 \%$ deprotonated, and STM reveals the presence of a new phase on the surface (shown schematically in Fig. 1c, with a typical STM image in Fig. 2a). We refer to the dominant bonding motif in this phase as "discrete pinwheel". The basic building block is a hexamer of TMA arranged around a bright central protrusion, outlined in white in Fig. 2a. The hexamer possesses an intrinsic chirality associated with a slight rotation $\left(\sim 20^{\circ}\right)$ of each TMA molecule with respect to the high symmetry directions of the hexagonal unit cell of the pinwheel structures. We interpret the hexamers as arising from the coordination of six $-\mathrm{COO}^{-}$groups around one or more silver adatoms, with the remaining twelve carboxylic groups around the outside of the pinwheel intact. This geometry provides a plausible hydrogen-bonding interaction between the intact-COOH groups at the perimeter of the pinwheel. Gas-phase DFT calculations at the B3LYP/6-31G(d,p) level (see ESI $\dagger$ ) suggest that a stabilization energy of $-2.2 \mathrm{kcal} \mathrm{mol}^{-1}$, which is substantially weaker (by a factor of 20) than per-molecule tabulated values for TMA in $\mathrm{R}_{2}^{2}(8)$ dimeric associations, ${ }^{30}$ but is nevertheless bound even in the gas phase. This hydrogen bonding interaction appears to propagate chirality, resulting in enantiomerically pure domains. The metal coordination at the centre of the pore is beyond the scope of our gas-phase DFT. However, the presence of one or more adatoms is implied through the brightness of the central protrusion across all bias voltages in STM imaging, and is consistent with the behaviour of deprotonated TMA in other surface-confined systems, where the formation of metal-organic phases is frequently observed. ${ }^{13,14,17}$ Furthermore, the O1s core level energetics suggest metal coordination, which has previously been shown to modify the observed binding energy of the carboxylate component (see ESI $\dagger$ ). ${ }^{31}$

In theory, the discrete pinwheel phase should correspond to a TMA deprotonation level of $33 \%$ (one $-\mathrm{COO}^{-}$per molecule). However, our XPS indicates a higher deprotonation level. This is consistent with the fact that, in several repeated experiments, we never observed pure-phase domains of discrete pinwheel. In all cases, the discrete pinwheel domains contained some molecules in a more advanced state of deprotonation (see below). This could
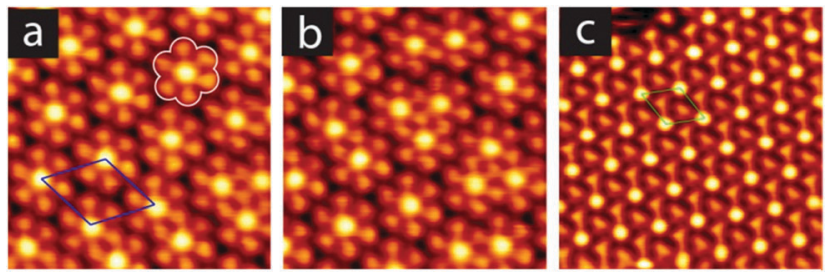

Fig. 2 STM images of (a) the discrete pinwheel phase, (b) the granular alloy mixed phase, and (c) the knitted pinwheel phase. A unit cell is drawn on the periodic structures in (a) (blue) and (c) (green), and a single pinwheel is outlined in white in (a). All images $8 \times 8 \mathrm{~nm}^{2}$ in size, (a) $i_{\mathrm{T}}=50 \mathrm{pA}$, $V_{\mathrm{B}}=-50 \mathrm{mV}$, (b) $i_{\mathrm{T}}=50 \mathrm{pA}, V_{\mathrm{B}}=-50 \mathrm{mV}$, (c) $i_{\mathrm{T}}=250 \mathrm{pA}, V_{\mathrm{B}}=-250 \mathrm{mV}$. 
be due to poor preparation parameters (consistently overshooting the desired phase), or to an intrinsic kinetic barrier to formation of the discrete pinwheel phase as a pure phase, e.g., if the nucleation of the domain requires the presence of some further-deprotonated structures.

Annealing to $495 \mathrm{~K}$ results in complete deprotonation of the molecule, as shown in Fig. 1d, where all spectral weight associated with $-\mathrm{COOH}$ has been lost, and the ratio of benzene carbon to $-\mathrm{COO}^{-}$falls slightly below 2:1 (see ESI $\dagger$ ). This chemical state of the molecule corresponds to a change in the observed structure, shown schematically in Fig. $1 \mathrm{~d}$ and in the STM image in Fig. 2c. This phase is structurally similar to the discrete pinwheel phase, but comprises an increased density of bright metal-atom centres, consistent with complete deprotonation of the TMA and the replacement of all hydrogen bonds with the metal-coordination geometry at the centre of the discrete pinwheel phase. We therefore refer to this phase as "knitted pinwheel". Like its companion phase, it possesses an intrinsic chirality related to a rotation of the individual TMA molecules with respect to the high-symmetry direction of the knitted pinwheel unit cell (shown in green in Fig. 2c. See ESI $\dagger$ for additional images demonstrating chirality).

Knitted pinwheels are present as a minority structure in all observed domains of the discrete pinwheel. At deprotonation levels near $50 \%$, the discrete pinwheel structure dominates. However, at deprotonation levels increasing from $50 \%$ towards $100 \%$ we find a continuum of hybrid domain structures where the discrete pinwheel phase coexists with an increasing concentration of knitted pinwheels. A typical STM image is included as Fig. 2b. The knitted pinwheel sub-domains vary in size, and lack long-range ordering. We therefore refer to this phase as a nonperiodic granular alloy. Like both the discrete and knitted pinwheel phases, this phase is chiral.

The seamless transition from discrete to knitted pinwheels suggests that the two phases have an intrinsic commensurability. Based on STM images where we could visualize the knitted pinwheel structure and the underlying $\operatorname{Ag}(111)$ lattice in the same frame, we can use image autocorrelation (see ESI $\dagger$ ) to assign the following epitaxy matrix:

$$
\left(\begin{array}{rr}
4 & -1 \\
1 & 5
\end{array}\right) \text {. }
$$

Based on this epitaxial constraint, we can use a patch of knitted pinwheel phase to project a lattice across an image that shows the nonperiodic phase; this type of analysis is shown in Fig. 3a. From this projection, we can see that the discrete pinwheel phase has an $8 \times 8$ unit cell that is aligned with the substrate lattice vectors. The analysis in Fig. 3b shows how the two phases relate to one another, and how the granular alloy formed from the two phases allows the accommodation of various chemistries. We also find that a sum of the expected deprotonation levels per unit area weighed by the density of the structures as shown in Fig. 3 leads to a mean deprotonation level of $50 \%$ over the whole surface, precisely in line with our estimate from the

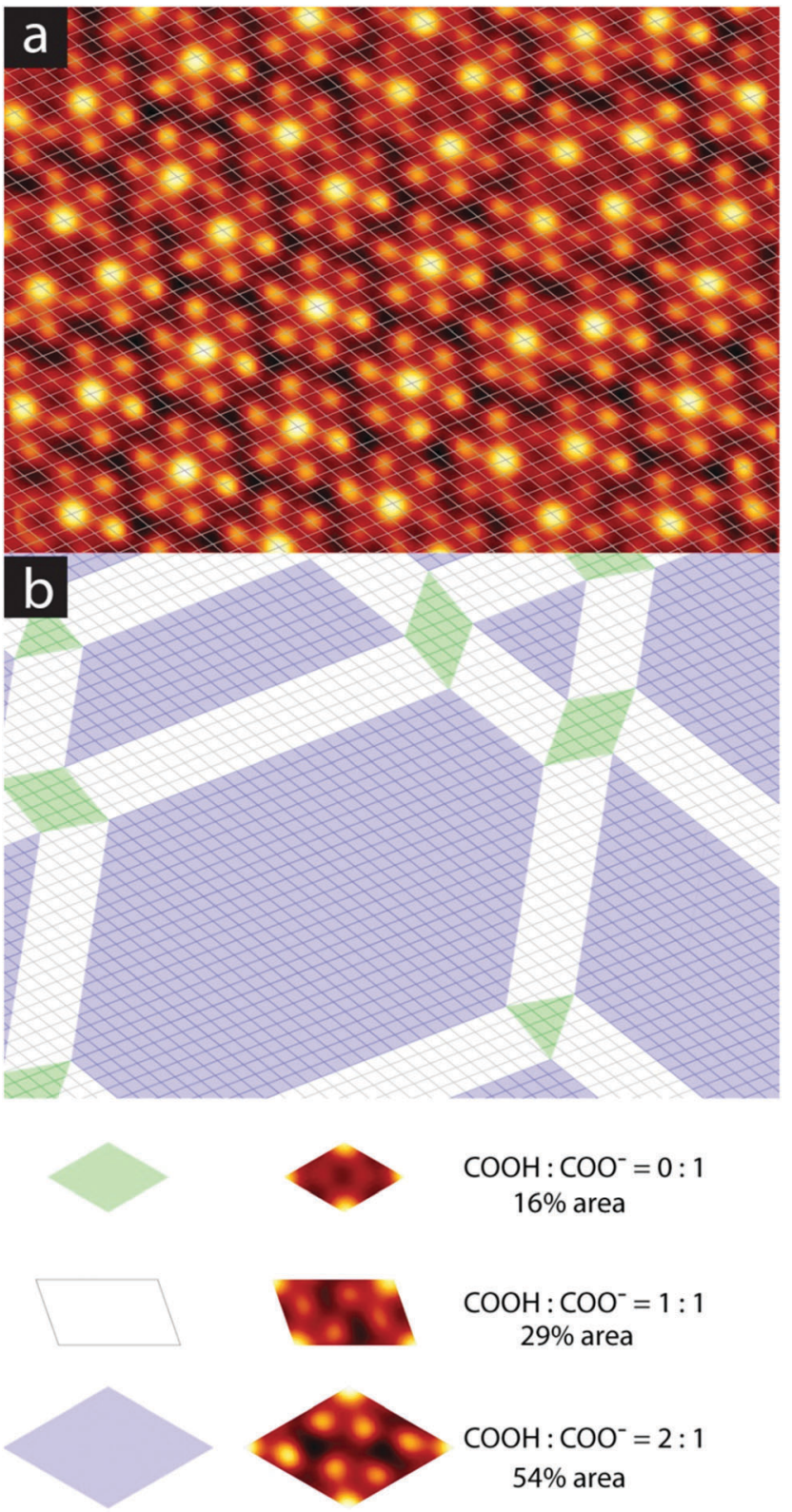

Fig. 3 Analysis of reacted state of the individual molecules in a granular alloy domain. (a) STM image, lattice-corrected to the discrete pinwheel phase. STM image parameters: $14.7 \times 10.4 \mathrm{~nm}^{2}, i_{\mathrm{T}}=10 \mathrm{pA}, V_{\mathrm{B}}=-110 \mathrm{mV}$. (b) Analysis of composition of the domain shown in (a), with colours assigned according to the legend at the bottom of the figure.

XPS measurements, and suggesting that the surface does not comprise regions of widely varying chemistry.

We have observed extended domains of this granular alloy phase. The image in Fig. 4 shows a $60 \times 60 \mathrm{~nm}^{2}$ image that showcases the seamless integration of the two sub-phases. The assigned epitaxy matrices indicate that there is a slight rotation between the packed and knitted pinwheel phases, and this is captured in the inset 2D-FFT, which shows that the three primary periodicities, associated with the discrete and knitted pinwheels and the individual molecules, are all rotated with respect to one another. This creates a chiral signature in the FFT. 


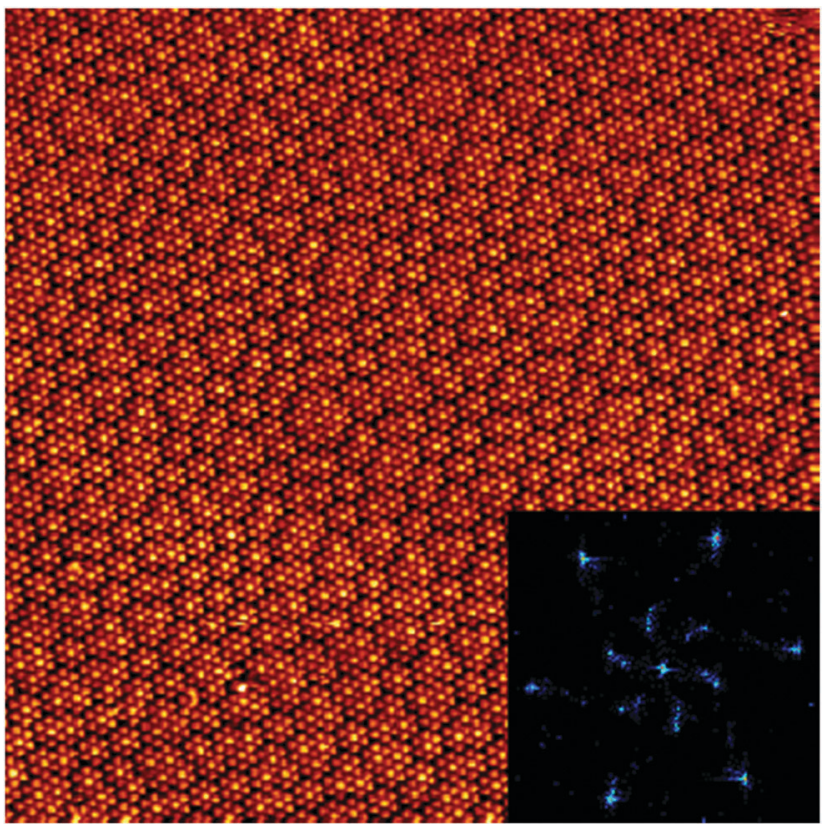

Fig. 4 Large-scale image of a granular alloy domain, with an inset 2D-FFT showing the rotations of the discrete and knitted unit cells with respect to the lattice defined by the individual TMA molecules. $60 \times 60 \mathrm{~nm}^{2}$ in size, $i_{\mathrm{T}}=300 \mathrm{pA}, V_{\mathrm{B}}=-1.3 \mathrm{~V}$.

This work demonstrates that a simple molecule can form the basis for complex, multi-interaction structures in a $2 \mathrm{D}$ selfassembled system. The nonperiodic phase defined by partially deprotonated TMA on Ag(111) demonstrates a number of interesting characteristics, including chirality and tolerance for a continuum of chemical states. The formation of this granular alloy phase is dependent on the geometrical compatibility of the epitaxial basis for the two phases, which emphasizes the important role of the substrate in this system. In future work we hope to elucidate more details of the metal-coordinated stabilization of the pinwheel phases, and to explore whether similar phases exist on other metal substrates or with other metals acting as the coordination centre.

Some of the data reported in this paper were obtained at the Central Analytical Research Facility (CARF) operated by the IFE at QUT. Computing resources and support were made available through QUT's High Performance Computing (HPC) centre. The authors acknowledge funding from the Australian Research Council through DE170101170 (JMM) and DP160103116 (JLD).

\section{Conflicts of interest}

There are no conflicts of interest to declare.

\section{Notes and references}

1 K. S. Mali, N. Pearce, S. De Feyter and N. R. Champness, Chem. Soc. Rev., 2017, 46, 2520-2542.

2 J. MacLeod and F. Rosei, Small, 2014, 10, 1038-1049.

3 M. Gobbi, S. Bonacchi, J. X. Lian, Y. Liu, X.-Y. Wang, M.-A. Stoeckel, M. A. Squillaci, G. D'Avino, A. Narita and K. Müllen, Nat. Commun., 2017, 8, 14767.

4 L. Sosa-Vargas, E. Kim and A.-J. Attias, Mater. Horiz., 2017, 4, 570-583.

5 R. Gutzler, L. Cardenas and F. Rosei, Chem. Sci., 2011, 2, 2290-2300.

6 K. Hipps and U. Mazur, Langmuir, 2017, 34, 3-17.

7 O. Ivasenko and D. F. Perepichka, Chem. Soc. Rev., 2011, 40, 191-206.

8 S. Griessl, M. Lackinger, M. Edelwirth, M. Hietschold and W. M. Heckl, Single Mol., 2002, 3, 25-31.

9 J. MacLeod, J. Lipton-Duffin, D. Cui, S. De Feyter and F. Rosei, Langmuir, 2015, 31, 7016-7024.

10 Q. Zhou, Y. Li, Q. Li, Y. Wang, Y. Yang, Y. Fang and C. Wang, Nanoscale, 2014, 6, 8387-8391.

11 V. Iancu, K.-F. Braun, K. Schouteden and C. Van Haesendonck, Langmuir, 2013, 29, 11593-11599.

12 Y. Ye, W. Sun, Y. Wang, X. Shao, X. Xu, F. Cheng, J. Li and K. Wu, J. Phys. Chem. C, 2007, 111, 10138-10141.

13 J. Barth, J. Weckesser, N. Lin, A. Dmitriev and K. Kern, Appl. Phys. A: Mater. Sci. Process., 2003, 76, 645-652.

14 N. Lin, A. Dmitriev, J. Weckesser, J. V. Barth and K. Kern, Angew. Chem., Int. Ed., 2002, 41, 4779-4783.

15 A. Dmitriev, N. Lin, J. Weckesser, J. Barth and K. Kern, J. Phys. Chem. $B, 2002,106,6907-6912$.

16 L. Kanninen, N. Jokinen, H. Ali-Löytty, P. Jussila, K. Lahtonen, M. Hirsimäki, M. Valden, M. Kuzmin, R. Pärna and E. Nommiste, Surf. Sci., 2011, 605, 1968-1978.

17 T. Classen, M. Lingenfelder, Y. Wang, R. Chopra, C. Virojanadara, U. Starke, G. Costantini, G. Fratesi, S. Fabris and S. De Gironcoli, J. Phys. Chem. A, 2007, 111, 12589-12603.

18 I. Cebula, H. Lu, M. Zharnikov and M. Buck, Chem. Sci., 2013, 4, 4455-4464.

19 H. Aitchison, H. Lu, R. O. de la Morena, I. Cebula, M. Zharnikov and M. Buck, Phys. Chem. Chem. Phys., 2018, 20, 2731-2740.

20 D. Payer, A. Comisso, A. Dmitriev, T. Strunskus, N. Lin, C. Wöll, A. DeVita, J. V. Barth and K. Kern, Chem. - Eur. J., 2007, 13, 3900-3906.

21 M. S. Baviloliaei and L. Diekhöner, Phys. Chem. Chem. Phys., 2014, 16, 11265-11269.

22 N. Lin, D. Payer, A. Dmitriev, T. Strunskus, C. Wöll, J. V. Barth and K. Kern, Angew. Chem., Int. Ed., 2005, 44, 1488-1491.

23 M. Gastaldo and S. Kiel, MSc thesis, Aalborg University, 2013.

24 Z. Zhou and K. D. Harris, ChemPhysChem, 2006, 7, 1649-1653.

25 N. A. Wasio, R. C. Quardokus, R. P. Forrest, C. S. Lent, S. A. Corcelli, J. A. Christie, K. W. Henderson and S. A. Kandel, Nature, 2014, $\mathbf{5 0 7}, 86$.

26 H. Zhou, H. Dang, J.-H. Yi, A. Nanci, A. Rochefort and J. D. Wuest, J. Am. Chem. Soc., 2007, 129, 13774-13775.

27 J. M. MacLeod, J. Lipton-Duffin, C. Fu, T. Taerum, D. F. Perepichka and F. Rosei, ACS Nano, 2017, 11, 8901-8909.

28 J. I. Urgel, D. Écija, G. Lyu, R. Zhang, C.-A. Palma, W. Auwärter, N. Lin and J. V. Barth, Nat. Chem., 2016, 8, 657.

29 I. Horcas, R. Fernández, J. Gomez-Rodriguez, J. Colchero, J. GómezHerrero and A. Baro, Rev. Sci. Instrum., 2007, 78, 013705.

30 J. M. MacLeod, O. Ivasenko, C. Fu, T. Taerum, F. Rosei and D. F. Perepichka, J. Am. Chem. Soc., 2009, 131, 16844-16850.

31 S. L. Tait, Y. Wang, G. Costantini, N. Lin, A. Baraldi, F. Esch, L. Petaccia, S. Lizzit and K. Kern, J. Am. Chem. Soc., 2008, 130, 2108-2113. 